\title{
A Measure of Risk Caused by Information Asymmetry in e-Commerce
}

This research is partially supported by NFSI-BG, Grant No VU-MI-105/2005

\author{
Dimitar Christozov \\ American University in Bulgaria, \\ Blagoevgrad, Bulgaria
}

dgc@aubg.bg

\author{
Stefanka Chukova \\ Victoria University of Wellington, \\ Wellington, New Zealand
}

Stefanka.Chukova@vuw.ac.nz

\author{
Plamen Mateev, \\ University of Sofia "St. Kliment Ohridski”, Sofia, Bulgaria \\ pmat@fmi.uni-sofia.bg
}

\begin{abstract}
The problem of enhancing success in e-Commerce requires reducing or sharing the risk of misinforming. The objectives of this paper is to raise and discuss the difficulties in quantifying the risk of misunderstanding caused by information asymmetry in a communication process; and to propose a mathematical model, developed to measure the risk of misinforming. Usage of warranty, as the most popular tool of sharing risk, is discussed in the light of risks of misinforming. A data set collected in an experiment, was used to illustrate the major ideas proposed as well as to stress on the fact that in particular circumstances this kind of risk is not understood by clients, which often leads to wrong decisions.
\end{abstract}

Keywords: information asymmetry, misinforming, warranty, risk

\section{Introduction}

The success of a business deal in e-commerce, involving the two parties - a seller and a buyer, depends on variety of factors, divided into two major groups:

o Environmental factors: properties of the environment in which the seller-buyer relationship operates, such as IT infrastructure, logistic infrastructure, financial infrastructure and government regulations.

Material published as part of this publication, either on-line or in print, is copyrighted by the Informing Science Institute. Permission to make digital or paper copy of part or all of these works for personal or classroom use is granted without fee provided that the copies are not made or distributed for profit or commercial advantage AND that copies 1 ) bear this notice in full and 2) give the full citation on the first page. It is permissible to abstract these works so long as credit is given. To copy in all other cases or to republish or to post on a server or to redistribute to lists requires specific permission and payment of a fee. Contact Publisher@InformingScience.org to request redistribution permission. o Communicational factors: properties of interpersonal relationship between the two parties, such as efficiency and effectiveness of the communication process, trust and confidence, content and acceptance of the exchanged messages, etc.

The information asymmetry, as a natural property of any communication process, 
may result in misunderstanding between sellers and buyers and therefore may erode the trust between them. This is critical especially in indirect communication between the two parties, as in e-commerce. From customer's point of view, missing of adequate knowledge about the product may result in wrong purchase decision. From point of view of the seller, customer's misunderstanding about the properties of the products may result in customer's dissatisfaction and loss of market positions. Often, the customer does not know exactly what s/he is buying and the seller doesn't know why the customer is buying the product. The two parties will benefit from overcoming the information asymmetry as well as from introducing techniques to evaluate and share the risk caused by the information asymmetry. Major instrument used in sharing risk is including a warranty statement into purchase contract.

\section{Information Asymmetry: Risks Caused by Misinforming}

\section{What is Information Asymmetry?}

In economics, information asymmetry occurs when one party to a transaction has more or better information than the other party. Also, it has been called asymmetrical information and related markets are called markets with asymmetrical information. Typically it is the seller that knows more about the product than the buyer, however, it is possible for the reverse to be true -- for the buyer to be better informed compare to the seller.

Examples, where the seller usually has better information than the buyer are numerous and include used-car salespersons, stockbrokers, real estate and life insurance agents. On the other hand, there are situations where the buyer is usually better informed than the seller, e.g., in insurance, when a buyer purchases insurance for a property with hidden problems.

The informational misbalance was described for the first time by Kenneth J. Arrow (1963), who introduces it as “moral hazard”. Later, George Akerlof (1970) used the term asymmetric information, noticing that, in a market with asymmetrical information, the average value of the commodity tends to go down, even for commodities with a perfect quality. Moreover, it is possible for such a market to decay to the point of nonexistence. Because of information asymmetry, unscrupulous sellers can embellish the items (like software or computer games) and defraud the buyer. As a result, many buyers with a risk-averse attitude will avoid certain types of purchases, or will not be willing to spend much on a given item.

Information Asymmetry exists in every communication process. Incorrect, not as intended, interpretation of the received message, as a side effect of the communication process, may cause significant qualitative and quantitave losses to both, the sender and the receiver. These side effects may be described as misunderstanding, misinterpretation, mis-inference, etc. Onwards, we will refer to all of these as misinforming. Reduction of losses caused by misinforming may be achieved either by improving the quality of the message sent or by introducing tools (as warranty) to share this risk between two parties. Sharing risk requires understanding the nature and quantifying the risk of misinforming. Evaluation of the risk caused by information asymmetry is especially important in indirect communication, such as e-commerce.

Quantifying the risks of malfunctioning of the products is relatively well studied (see for example Blischke \& Murthy, 1993, 1996; Chukova, Arnold, \& Wang, 2004). It uses models, based on information obtained from reliability tests, quality control or other activities carried out by the producer to study product's performance. The comprehensiveness and precision of this assessment is entirely under the control of the producer and the assigned warranty policies fully reflect the objectives of the producer.

On the other hand, measuring the risk of misinforming is based on the failures in the communication process. It is not under the contol of any of the parties involved in the 
communication and it requires different approaches and new techniques for collecting and processing the relevant data. The primary data source is the customer's feedback on the satisfaction with the product. Studies on the success of information services originate in the area of Information Sciences (see Buckland 1991, page 173), where, in a relatively narrow settings, the impact of a receiver's expertise on the success of the information services was studied. The reciever's expertise was studied by Buckland in the two aspects - subject expertise and expertise to use efficiently the information system providing the servce. The impact of receiver's expertise on both aspects, as defined by Buckland, are of crucial importance for the success in e-commerce. Lack of expertise in using the information system simply excludes these customers from the market. The level of receiver's expertise in the subject area affects the level of information asymmetry - a high expertise in the subject area reduces the level of information asymmetry and vice versa.

\section{The Nature of Misinforming}

A communication process represents an exchange of information between two parties - a sender and a receiver - throught a given communication channel. The stages in a communication process are represented in Figure 1.

The sender sends out a message addressing the receiver. The content of the message is a sign of the sender's idea of what is useful to the receiver. The message is composed to represent the meaning as it is understood by the sender. Its structure is influenced by the sender's judgment on the receiver's capability to understand and interpret the message correctly. The message is encoded to make it suitable for transmission through the communication channel and submitted to the receiver.

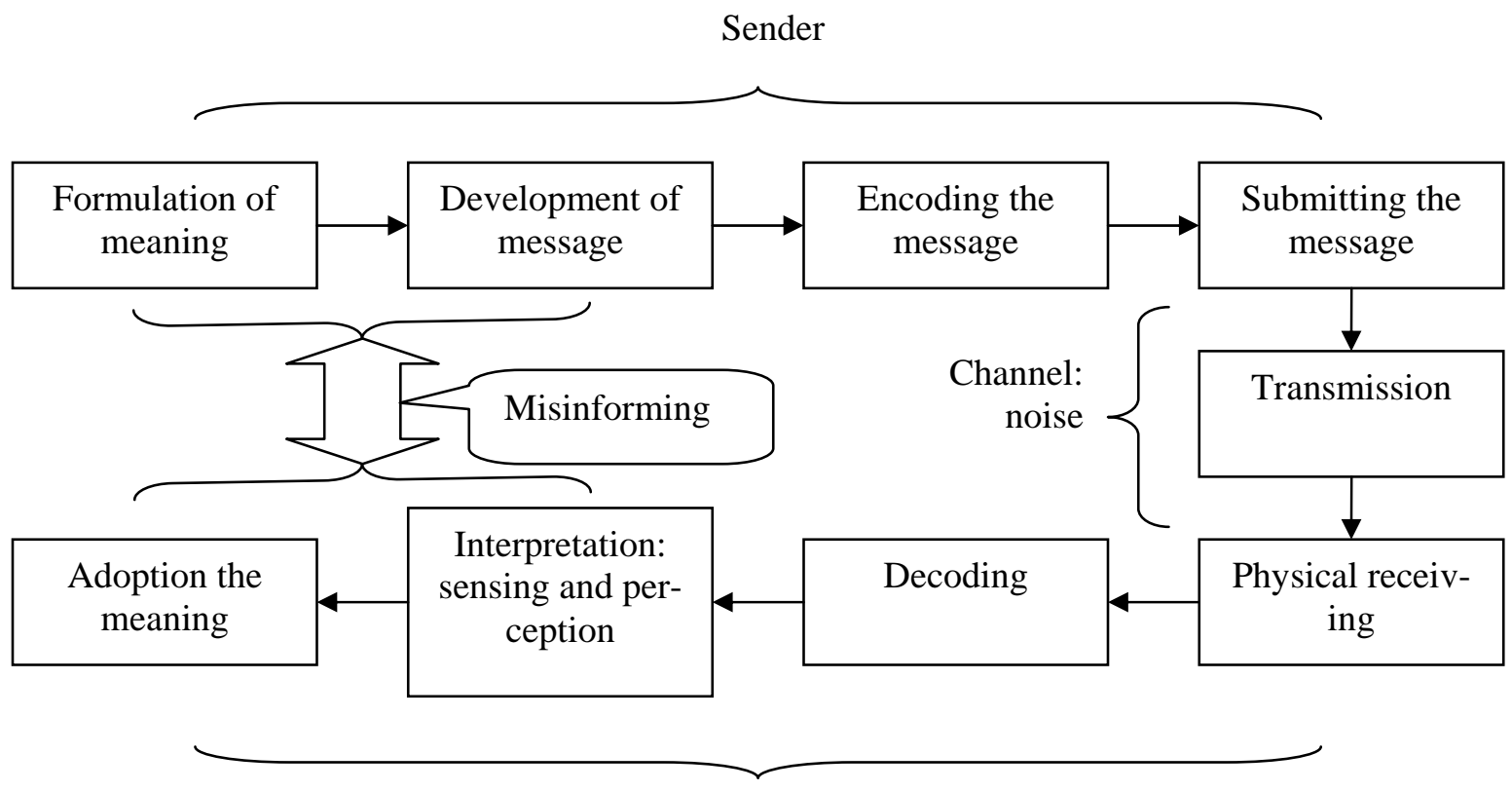

Receiver

Figure 1. Stages in a communication process

From the receiver's side, the process also includes several stages. It starts with the physical delivery of the message followed by its decoding. The decoding is the ability of the receiver to read the message, which requires an agreement between the two parties on the language used in 
the message. The understanding, i.e., the proper interpretation of the message is essential for the success of the communication process. The last phase - adoption of the information is successful if the receiver utilises the obtained information in making decisions. It depends on the succes of all of the previous stages in the communication process. Failure in any of them results in a failure in the communication.

Most of the technological tools developed to support the communication are focusing on the physical delivery and correct decoding of the information.

The stage of interpretation the content of the message is critical to whether the message informs or misinforms the receiver. The interpretation is based on receiver's expertise, perceptions, biasness, etc. The success in this stage is seriously influenced by the information assymetry defined as the difference in expertise of the sender and reciever on the subject of the message.

The last stage, the adoption of the information, depends on whether the obtained information, as it is understood, is valuable to the receiver. Also, it is greatly influenced by the level of sender's credability with the receiver. In a face-to-face communication, the trust may be revealed by many factors additional to the content of the message, such as whether the communication takes place in a trustful environment, what is sender's personality, sender's body language, possibilities of clarifying the meaning of the message in an easy and natural dialog, etc. In indirect communication, majority of these factors are irrelevant and they have to be compensated by the structure and the content of the message.

\section{Issues and Possible Solutions}

There are many issues and problems naturally arising within an indirect communication environment and, as expected, reflect on the success in e-commerce. Some of these issues are:

1. Whether the meaning of the message intended by the sender matches the meaning understood by the receiver?

2. What is the risk and related business effects due to a wrong interpretation of the meaning of the message from a receiver's point of view as well as from sender's point of view?

3. How should a communication process be modified in order to increase the chance for success?

Better results in an indirect communication process could be achieved by reducing the risk of misinforming for the receiver and by encouraging him/her to utilise the information received.

The reduction of the risk of misinforming may be accomplished in two ways:

- Reducing the information assymetry, by increasing the level of mutual understanding between the two parties.

- Offering a warranty to cover the risk of misinforming, by sharing the risk between the two parties.

Both approaches require collecting and analyzing data related to the feedback on the success of an indirect communication process.

Techniques like Customer Related Management (CRM) or Market Studies address the first of the two approaches. The study of the feedback within a certain commercial activity increases the seller's understanding about the client's expertise. It allows them to compose their messages to target the specifics in the client's backgrounds. Market Studies define groups of customers with similar needs and interests and allow sellers to address the group as a whole at an appropriate 
level of complexity. CRM allows companies to offer individually customised products and address the clients with specifically designed messages.

Warranty that includes statements like "Money back guarantee if not fully satisfied" is an attempt to solve the problem using the second approach, i.e., to increase the credibility of the seller. This type of warranty promotes:

- $\quad$ an increase in the customer's understanding about the product, by encouraging him/her to experiment with a limited risk,

- an increase in the producer's understanding of customer's language and allows for improvement in communications between the two parties in the future.

This type of warranty provides learning opportunities for both parties. It decreases the information asymmetry and enhances the trust between them. Unfortunately, in the case of indirect communication, the probability of misinforming can be high enough to cause large unexpected losses for the warrantor. Therefore, while designing reasonable warranty coverage, quantifying the risk of misinforming is a significant and often critical task.

\section{Quantitative measures}

Next, our goal is to develop a quantitative measure to assess the risk of information asymmetry. We start by introducing certain formalism.

Assume that a particular customer needs to solve a set of problems, say $\{A\}$. Subsequently, we will refer to this customer as a customer with set $\{A\}$. The elements $w$ of $\{A\}$ are disjoined, called tasks, and

$$
\{A\}=\left\{w_{i}, i=1, \ldots N\right\},
$$

where $N$ is a positive integer and $N<$ infinity.

Also, let us assume that by applying certain methods, devices or services, a task $w_{i}$ from $\{A\}$ can be solved. We will call these methods, devices or services products. Moreover, we assume that there is a set of products $D=\left\{d_{j}\right\}, j=1, \ldots, M$, available on the market, appropriate for solving the tasks in $\{A\}$.

For different customers, who have the same set of tasks $\{A\}$, the complexity and importance of tasks varies and, correspondingly, the suitability of the products, available for solving them, also varies. We assume that for each set of tasks $\{A\}$ and each particular customer with set $\{A\}$ a specific threshold of acceptance $q, 0<=q<=1$, exists and its level (value) is pre-set by the customer. The threshold of acceptance is the lowest level, set by the customer, of product's suitability still acceptable for solving the set $\{A\}$. The threshold of acceptance represents the customer's opinion on how suitable the product should be in order to be considered as a possible solution for the tasks in $\{A\}$. The value of $q$ is independent of the set of products $D$.

The set of tasks $\{A\}$ can be considered as a sample space of all possible outcomes for a particular customer. Let $P=P(w)$ denotes a probability distribution on $\{A\}$, induced by the customer with set $\{A\}$. This probability distribution is subjective and represents the importance of the tasks to the customer.

Next, our aim is to define the probability that a given product is suitable for a customer with set $\{A\}$. Denote this event as "success" and mark it by $S$ an event. Let $P(S \mid w)$ be the probability 
that the customer can solve the task $w \in A$ without applying any product. Then, based of the total probability rule, the probability that the customer will successfully solve the set of tasks $\{A\}$ without applying any product is

$$
P(S)=\sum_{w \in A} P(S \mid w) P(w)
$$

Further, let us apply the product $d$ from $D$ for solving the task $w \in A$. Now, the probability of success is $P(S \mid w, d)$, which is a measure of the suitability of the product $d$ to solve task $w$. The product $d$ is suitable for solving $w$ if and only if

$$
P(S \mid w)<P(S \mid w, d),
$$

i.e., the probability to solve the task $w$ with product $d$ is higher then the probability of solving the task without applying any product. We will assume that this inequality holds for product $d$ and at least one of the tasks $w, w \in A$. Otherwise the product $d$ is irrelevant to the needs of the customer with set $\{A\}$.

The suitability of product $d$ for a customer with set $\{A\}$ can be measured with the probability of success when applying this product to all tasks $w \in A$, i.e.,

$$
p=P(S \mid d)=\sum_{w \in A} P(S \mid w, d) P(w) .
$$

Therefore, if a satisfactory estimation for the aforementioned probability is available it can be used to measure the suitability of product $d$ for set $\{A\}$.

The information asymmetry is due to a mismatch between the seller's and the buyer's estimations of the suitability of product $d$ with respect to a set $\{A\}$. The seller is familiar with the product and its suitability to solve a particular task, whereas the seller is better informed on the suitability of the product related to the tasks from $\{A\}$ measured by the probability $P(S \mid w, d)$.

Moreover, the customer is the only one who knows his/her own problems/tasks $\{A\}$ and related distribution $P(w)$. Therefore a customer is identified by the pair $\{(A, P), q\}$, the set of task s/he needs to solve, the measure of the importance of the tasks and the threshold of acceptance for set $\{A\}$. The triple $\{(A, P), q, d\}$ describes how a product $d$ meets customer's needs $q$ in solving tasks $W$ from given $(A, P)$.

Any message from the seller to the customer aims to enrich the customer's information about the product, which will reflect on the value of $P(S \mid w, d)$.

A customer makes his/her purchase decision based on the estimation $\hat{p}$ of $p=P(S \mid d)$, where $p$ given by (1). The estimation $\hat{p}$ summarizes the customer's understanding regarding the suitability of the product to solve his/her set $\{A\}$. In general, there are three categories of customers, optimists with $\hat{p}>p$, pessimists with $\hat{p}<p$ and realists with $\hat{p}=p$. The probability of having $\hat{p}=p$ is negligible, because it refers to exact equality of these two probabilities. In case we refer to realists as those for which $|\hat{p}-p|<\varepsilon$, where $\varepsilon^{\varepsilon}$ is a small positive - the difference is negligible, the group of realists deserve attention, but in this case we cannot speak about existence of information asymmetry. That's why we will not discuss the category of realists any further. 
The purchase decisions depends on the comparison between $q$ and $\hat{p}$. If $q<\hat{p}$, then the purchase decision should be positive, i.e., customer's estimation of the suitability of the product is greater than the threshold of acceptance and the product is assessed as suitable. If $q>\hat{p}$, the purchase decision should be negative, because customer's estimation of the suitability of the product is less than the threshold of acceptance and the product is not suitable for solving for $\{A\}$. There are six possible scenarios, caused by the information asymmetry:

1. $\quad p<\hat{p}<q$ - the product is not suitable, the customer's estimation of the suitability of the product is optimistic and below the threshold of acceptance, thus the decision is negative and correct;

2. $\quad p<q<\hat{p}$ - the product is not suitable, the customer's estimation of the suitability of the product is optimistic and above the threshold of acceptance, thus the decision is positive and wrong;

3. $q<p<\hat{p}$ - the product is suitable, the customer's estimation of the suitability of the product is optimistic and above the threshold of acceptance, thus the decision is positive and correct;

4. $\quad \hat{p}<p<q$ - the product is not suitable, the customer's estimation of the suitability of the product is pessimistic and below the threshold of acceptance, thus the decision is again negative and correct;

5. $\hat{p}<q<p-$ the product is suitable, the customer's estimation of the suitability of the product is pessimistic and below the threshold of acceptance, thus the decision is negative and wrong;

6. $q<\hat{p}<p$ - the product is suitable, the customer's estimation of the suitability of the product is pessimistic and above the threshold of acceptance, thus the decision is positive and correct.

The first three cases specify the customer as a pessimist and the last three - as an optimist. In each of these categories a wrong decisions is possible. For optimists, the wrong decision is in case 2 - the product is not suitable, but the purchase decision is positive, whereas, for pessimists, the wrong decision is in case 5 - the product is suitable, but the purchase decision is negative.

\section{An Illustrative Example}

Next, in order to illustrate the ideas, we consider an example related to the evaluation of the risk of misinforming by selling/buying a personal computer. Usually, the information provided by the seller, regarding the configuration of the system is presented in the following form:

Pentium IV/1.6C GHz
Intel 440 - LX Power Pro ATX
256MB DDR RAM
1.44 MB FDD
30GB HDD QUANTUM
16MB SGRAM VGA card AGP
15' Color HANSOL 0.28, LR
N.I. (1024x768), CPU CONTROL

On the other hand, usually the buyer is looking for information, regarding the capabilities of the systems to (set of tasks $\{A\}$ ): 
- develop and edit text;

- perform high precision computations;

- use of e-mail;

- $\quad$ easy access to the Internet;

- $\quad$ organize personal data;

- play games;

- watch movies.

In addition, the seller offers warranty coverage, aiming to increase his credibility by sharing the risk of malfunctioning of the computer as well as the risk of misinforming the customers. There are two types of warranty statements and they address the two different sources of the risk.

The standard warranty statement consisting something like "All computers (battery/power adapter excluded) are covered for a period of 1 year warranty from the date of original purchase, except in cases where the manufacturer's warranty exceeds ours. In these cases the manufacturer's warranty will be honored" (see http://www.rjtech.com/notebook warranty.htm). It addresses the risk caused by the low reliability of the hardware and this is a warranty on the defectiveness and malfunctioning of the item.

On the other hand, the buyer making his/her purchase decision is interested in the following questions:

- What is the meaning of ATX, AGP, etc.?

- Whether this configuration can fit his/her needs in terms of software and computing capabilities?

- Whether s/he needs all of the listed components of the system? and so on.

A warranty statement consisting something like "We guarantee 30-day money back for most items, except CPU and memory are 7-day money back. The exception for the CPU and memory is necessary due to the frequent price fluctuation in these items", (see http://www.directron.com/money-back-guarantee-.html ) addresses the risk of miscommunication, misunderstanding, misinforming between the seller and the buyer. In this case, the buyer has an easy task in making his/her decision, because the warranty provides a full protection against any wrong decision for a certain period of time. Also, this type of warranty strongly promotes the sales of the warranted product.

Next, we discuss an illustrative numerical example on evaluation of the risk of misunderstanding. We have designed a simple experiment, collected the relevant data and used them to evaluate the risk of misunderstanding. The original design of the experiment, description of the collected data and their aggregation are described in Christozov and Mateev 2003. The same experiment was repeated twice since Fall 2002 and combined results are presented in Table 1. Designed to illustrate before students the problem of misinforming, the original test did not match perfectly to the need of the current study, but to achieve comparability in repeating experiments we decided to use the same construction of the test, nevertheless that we set further more clearly the objectives of this study.

Target group: a group of sixty students (35 responded), mostly sophomores, were offered to purchase a personal computer (PC). We assumed that these students have a good understanding about their needs regarding the usage of a personal computer, because they actually are facing purchasing computers. The data were collected during the Fall semester of 2002.

The message: the target group was approached with an offer to buy a PC, with powerful computational capabilities, designed to serve professional scientists. It had no communication devices; 
no color graphics and the sound capabilities were limited. The offer was presented in the following form:

\section{ASUS A7V333/RA sA, VIA KT333,266,ATA 133,RAID, 3DDR,AGP 4x, USB 2.0, IEE 1394, ATX AMD XP PALOMINO 2100PR/1.75 GHz DDRAM 512 MB DDR, PC 333MHz, SAMSUNG 80 GB MAXSTOR / 7200 / ATA 133/2MB PCI ATI 1MB 17' SAMSUNG 753S 1280x1024/65Hz 1024x768/85 Hz BW keyboard, mouse, midi tower case}

The offered PC were designed for word processing and complex computations, but not for the use of the Internet and e-mailing, did not have any capabilities for multimedia, playing games, watching movies, etc. The price was realistic, but highly attractive.The survey

1. The students were asked to evaluate whether the given PC satisfies their needs, according to the following list of applications:

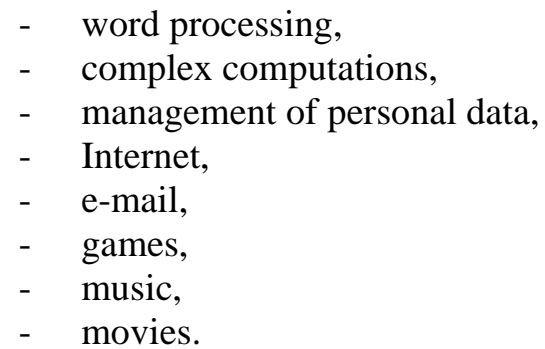

The students had to provide two answers for every item of this list, firstly, whether they need the given application and, secondly, whether the given PC allows for such an application.

2. They were offered two warranty statements: either "Three years standard warranty" or "Three months "money back guarantee" warranty" and were asked to make their purchase decision.

Table 1. Summary of survey results

\begin{tabular}{|c|c|c|c|c|c|c|}
\hline Application & \multicolumn{2}{|c|}{ Needs } & \multicolumn{2}{|c|}{ Assessed ability } & Actual ability & Wrong decision \\
\hline 1 & \multicolumn{2}{|c|}{2} & \multicolumn{2}{|c|}{3} & 4 & 5 \\
\hline$A=\{w\}$ & \# & $P(w)$ & \# & $\hat{p}$ & $p$ & $a b s(p-\hat{p})$ \\
\hline Word Processing & 33 & 0.143 & 27 & 0.77 & 1.00 & 0.23 \\
\hline Complex Computations & 10 & 0.043 & 19 & 0.54 & 1.00 & 0.44 \\
\hline e-mail & 35 & 0.152 & 25 & 0.71 & 0 & 0.71 \\
\hline Internet & 35 & 0.152 & 25 & 0.71 & 0 & 0.71 \\
\hline Maintain Personal data & 33 & 0.143 & 27 & 0.77 & 1.00 & 0.23 \\
\hline Play Games & 22 & 0.095 & 21 & 0.60 & 0 & 0.60 \\
\hline Movies & 28 & 0.121 & 21 & 0.60 & 0 & 0.60 \\
\hline Music & 35 & 0.152 & 20 & 0.57 & 0 & 0.57 \\
\hline Overall Risk: & & & & & & 0.51 \\
\hline
\end{tabular}

The column "Applications" represents the set of tasks $\{A\}$. The column "Needs" consists of subjectively defined needs, which are proportional to the estimates of the probabilities $P(w)$. The 
third column shows the subjective estimate of product's suitability (expressed by $\hat{p}$ ) with respect to the tasks as seen by the customers. The fourth column consists of $p=P(S \mid w, d)$, the objective ability of the product to solve the task. The last column shows the share of customers who may do wrong decision, based on misunderstanding the ability of the product to solve the given task, which is computed using the approach discussed on Section 3. In this case $p$ is either 1 or 0 and the threshold $q$ does not play any role, because if we consider a particular $w_{i}$, we have the following cases:

1) if $p=0$, the product is totally unsuitable, then both $q$ and $\hat{p}$ are greater or equal than $p$ and any positive purchase decision is wrong;

2) if $p=1$, the product is absolutely suitable and both $q$ and $\hat{p}$ are less or equal than $p$, and any negative purchase decision is wrong.

The risk caused by misunderstanding is calculated as follows: The risk of a wrong decision caused by the information asymmetry is equal to $r=a b s(p-\hat{p})$ and depends on $p$ - the objective suitability of the product, described by the seller in the message (seller's information), and on $\hat{p}$ - the suitability of the product as the buyer understands and interprets the message (buyer's information). For the i-task ( $\left.w_{\mathrm{i}}\right)$ the measure of its risk caused by information asymmetry is either $r=(p-\hat{p})$ if $p=1$ and the product is suitable, but purchase decision is negative, or $r=(\hat{p}-p)$ if $p=0$ the product is unsuitable, but purchase decision is positive.

The overall risk of a wrong decision due to misinforming is evaluated by the weighted average of the information asymmetries of the tasks, where the weights set to be equal to the corresponding "needs" $P(w)$, i.e.,

$$
R=\sum P\left(w_{i}\right) r_{i}
$$

The overall risk of misinforming turned out to be a relatively high for this particular case. This could be due to a number of reasons such as a low level of expertise of the buyers (students) regarding computer configurations, lack of easy to read and informative description of the components of the computer system and so on.

Finally, a great majority of students responded positively to the warranty statement addressing malfunctioning of the computer, and lower number to warranty allowing to experiment with the computer and to postpone the final decision until gaining confidence in suitability of the product.

\section{Conclusion}

The findings of our study can be summarized in the following way:

The risk caused by information asymmetry could be high enough to incur serious business consequences and it is important to be studied, understood and quantified. A significant percentage of customers do not understand the risk caused by misinforming. The customer's confidence about his/her ability to understand correctly a certain message is often overestimated, which usually leads to poor decisions.

2. E-commerce offers great opportunities for enriching the seller-customer relationship. An indirect business communication emphasizes the trust between the two parties. It requires development of tools to enhance the understanding between them and to overcome the information asymmetry.Sellers/Producers aiming to promote their products by covering the risk of misinforming, usually assume a negligible impact of 
this risk on their business. The learning aspects of warranty related to misinforming are underestimated as well. Warranty is a mechanism for sharing the risk between the seller and the buyer. On one hand, the seller receives customer's feedback regarding this risk and, on the other hand, it allows for an increase of customer's understanding about the product, by encouraging them to experiment with a limited risk. Growing customer's awareness and requirements towards quality in the marketplace forces producers/sellers to pay special attention to the risk of misinforming and to develop and apply techniques for the evaluation and sharing of this risk, similarly to what has been done for the risk of malfunctioning.

\section{References}

Akerlof, G. A. (1970). The market for 'lemons': Quality uncertainty and the market mechanism. Quarterly Journal of Economics, 84(3), 488-500.

Arrow K. (1963). Uncertainty and the welfare economics of medical care. AER.

Blischke W., \& Murthy, D.N.P. (1993). Warranty cost analysis. Marcel Dekker.

Blischke W., \& Murthy D.N.P. (1996), Product warranty handbook. Marcel Dekker.

Buckalnd, M. (1991). Information and information systems. NY: Preager.

Christozov, D., \& Mateev P. (2003). Warranty as a factor for e-commerce success. Proceedings of Informing Science and IT Education Conference, Pori, Finland, June 2003. Available at http://proceedings.informingscience.org/IS2003Proceedings/docs/068Chris.pdf

Chukova, S., Arnold, R., \& Wang, D. (2004) Warranty analysis: An approach to modelling imperfect repairs. International Journal of Production Economics, 89(1), 57-68.

\section{Biographies}

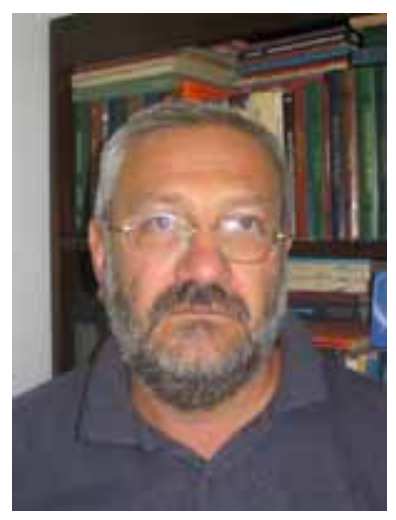

Dimitar Christozov is a Professor of Computer Science at the American University in Bulgaria, Blagoevgrad 2700, Bulgaria since 1993. He has more than 25 years of experience in areas as computer science, quality management and information systems. He graduated Mathematics from Sofia University "St. Kliment Ohridski” in 1979. He completed his PhD thesis "Computer Aided Evaluation of Machine Reliability” in 1986. In ICTT “Informa” (1986-1993) Dr. Christozov was involved in establishing the national information network for technology transfer and research in the areas of technologies assessment, integral quality measures and information systems for quality management. In these areas he was recognized as one of the leading experts in Bulgaria. Professor Christozov has more than 50 publications as separate volume, journal papers and papers in refereed procideengs. He is a founding member of Informing Science Institute and chair of Bulgarian Informing Science Society; and member of the Bulgarian Statisyical Society. 


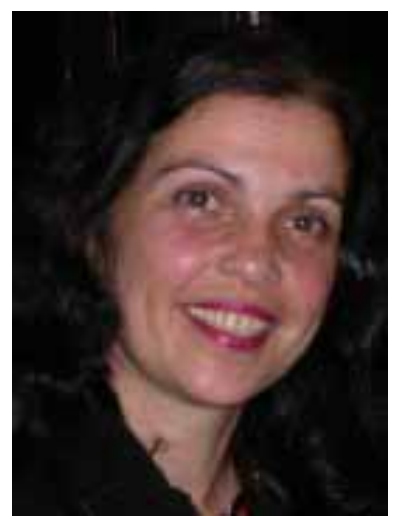

Dr. Stefanka Chukova is a Reader in Statistics and Operations Research at the School of Mathematics, Statistics and Computer Science, Victoria University of Wellington, Private Bag 600, Wellington, New Zealand, e-mail: schukova@mcs.vuw.ac.nz. She has a PhD and MSc in Mathematics (concentration in Probability and Statistics) and BSc in Mathematics from University of Sofia, Sofia, Bulgaria. Her research interests are in applied stochastic models, warranty analysis, reliability and queueing. She has more than 50 publications and has presented papers at national and international conferences and workshops. She is a member of ORSNZ, AWIS and ASA.

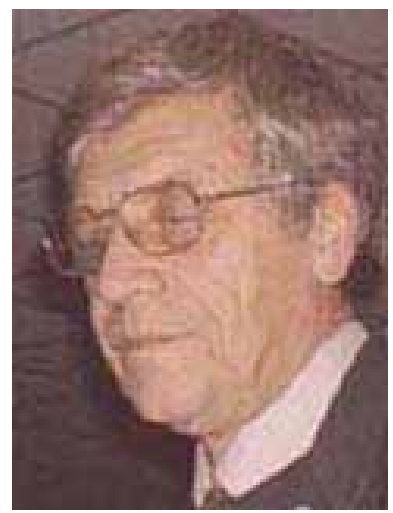

Dr.Plamen S. Mateev is Associate professor in Faculty of Mathematics and Informatics, Sofia University "St.Kliment Ohridski", Department "Probability, Operation research, Statistics", Bulgaria, 1164 Sofia, 5, J.Boucher str., e-mail: pmat at fmi.uni-sofia.bg.

His MSc in Mathematical Statistics is from Sofia University and his $\mathrm{PhD}$ is from Moscow State University. The research interests are in communication theory, applied statistics, statistical software and applications. More than 70 papers are published in scientific journals and proceedings of scientific conferences. He is a member of Bulgarian Statistical Society , ENBIS and InSITE Bulgaria.. 